Für Ärztinnen und Ärzte ist Achtsamkeit gegenüber dem eigenen Wohlbefinden und der eigenen Gesundheit kein luxuriöser Selbstzweck. Sind sie überlastet und unzufrieden, wirkt sich dies auch auf die Patientenversorgung aus: Gestresste Ärzte machen eher Fehler und überzeugen auch nicht als Vorbilder, kranke Ärzte kommen zudem teuer zu stehen! Sich um die eigene Gesundheit zu kümmern ist für Ärztinnen und Ärzte nicht nur ein Gebot der Selbstfürsorge, es ist ebenso eine Investition in die Qualitätssicherung und die Ökonomie des Gesundheitswesens.

Dr. med. Christine Romann, Mitglied des Zentralvorstandes der FMH, Departementsverantwortliche Gesundheitsförderung und Prävention

\title{
Doctors' health matters
}

\author{
Barbara Weil \\ Leiterin Abteilung Gesundheitsförderung und Prävention
}

Untersuchungen im In- und Ausland belegen, dass Ärztinnen und Ärzte in ihrer täglichen Arbeit erhöhtem psychosozialem Stress ausgesetzt sind. Der Wandel der Arbeitsbedingungen hat zur Folge, dass sie sich zunehmend ausgebrannt fühlen. Trotzdem identifizieren sich Ärzte nach wie vor sehr mit ihrer Tätigkeit und leisten gute Arbeit - was Öffentlichkeit und Patienten auch anerkennen. Gleichzeitig ist klar, dass sich etwas ändern muss, sonst wird die berufliche und private Lebensqualität vieler Ärztinnen noch stärker beeinträchtigt.

\section{Stress und Druck kennzeichnen heute vielfach den Berufsalltag von Ärztinnen und Ärzten.}

Auf Konferenzen zu Physicians' Health suchen Experten aus zahlreichen Ländern nach Wegen, die Gesundheit von Medizinern zu erhalten oder zu verbessern. Insgesamt wird vielfältige Unterstützung vom Medizinstudium bis zur Pensionierung angeboten: So existieren beispielsweise edukative, konfrontative und auch qualitätssichernde Angebote. Und ebenso können Ärzte eine Therapievermittlung oder ein Coaching in Anspruch nehmen. Angehende Mediziner werden während des Studiums und der Assistenzarztzeit auf einen besseren Umgang mit beruflichem Stress vorbereitet. Als Reaktion auf die Not vieler niedergelassener Ärzte gibt es TelefonHotlines, die schnelle und kompetente Hilfe vermitteln. Präventionsmassnahmen werden mithilfe von Informationsbroschüren, Gesundheitsprogrammen für Mitarbeitende von Kliniken oder Seminaren für Ärzte zum Thema «Burnout» realisiert. Aufklärung findet statt über das erhöhte Suizidrisiko von Ärzten und insbesondere Ärztinnen sowie über verfügbare Hilfen gegen Depression und Suizid. Ärzte mit Abhängigkeitsproblemen werden auf ihre Probleme ange- sprochen und in arztspezifische teilstationäre Therapien vermittelt.

\section{Effektive und praxisnahe Hilfsangebote}

Der Grad der Vertraulichkeit ist hoch. Manche Angebote via Internet, Telefon-Hotline oder Kurse können ohne Namensnennung genutzt, andere unter vier Augen wahrgenommen werden; denn die Schwelle bei Ärzten, sich Hilfe zu suchen, ist sehr hoch. Indiskretion, Rufschädigung und Blossstellung werden so sehr befürchtet, dass selbst schwer depressive und suizidale Ärzte oft so lange warten, bis es zu spät ist. Diese Fakten müssen Hilfssysteme berücksichtigen, die auf Ärzte zugeschnitten sind - andernfalls bleiben sie wirkungslos.

Von einem solch vernetzten präventiven Hilfssystem ist man in der Schweiz noch weit entfernt, auch wenn es verschiedene positive Ansätze gibt, etwa ReMed oder das «bureau des médecins de demain» des CHUV, die Interventionen des VSAO zur Einhaltung des Arbeitsgesetzes oder für familienfreundliche Mass-

Gesunde berufliche Rahmenbedingungen sind für eine hohe Patientensicherheit ebenso notwendig wie entsprechende Hilfsangebote.

nahmen seitens Arbeitgeber (in Zusammenarbeit mit der Fachstelle UND), Kinderkrippenvermittlung, oder das Label «Friendly Workspace» der Stiftung Gesundheitsförderung Schweiz.

Prävention und Gesundheitsförderung für Ärzte sollte zudem auch Eingang in die ärztliche Weiter- und Fortbildung finden. Die Gesundheit von Ärzten zu fördern und $\mathrm{zu}$ erhalten ist eine Notwendigkeit - sie dient der Behandlungsqualität und letztlich der Patientensicherheit. Die FMH will ihren Beitrag dazu leisten. 